\title{
Eficacia y seguridad de una vacuna contra la leptospirosis humana en Cuba
}

\author{
Raydel Martínez,, ${ }^{1}$ Alberto Pérez, ${ }^{2}$ María del C. Quiñones, ${ }^{3}$ Raúl Cruz,, \\ Ángel Álvarez, ${ }^{1}$ Marlén Armesto, ${ }^{2}$ Carmen Fernández, ${ }^{1}$ Jorge Menéndez, ${ }^{2}$ \\ Islay Rodríguez, ${ }^{1}$ Morelia Baró, ${ }^{2}$ Manuel Díaz,, José Rodríguez, ${ }^{1}$ \\ Gustavo Sierra, ${ }^{2}$ Ana Margarita Obregón, ${ }^{1}$ María Eugenia Toledo ${ }^{1}$ \\ y Norma Fernández ${ }^{3}$
}

Forma de citar

Martínez R, Pérez A, Quiñones M del C, Cruz R, Álvarez A, Armesto M, Fernández C, Menéndez J, Rodríguez I, et al. Eficacia y seguridad de una vacuna contra la leptospirosis humana en Cuba. Rev Panam Salud Publica. 2004;15(4):249-55.

RESUMEN Objetivos. Evaluar la eficacia de la vacuna cubana contra la leptospirosis vax-SPIRAL y aportar información adicional acerca de la seguridad de esta vacuna.

Métodos. Ensayo de eficacia (fase III) controlado, aleatorizado y con doble enmascaramiento de la vacuna cubana contra la leptospirosis vax-SPIRAL (Instituto Finlay, Cuba). Como control se utilizó la vacuna recombinante contra la hepatitis B Heberbiovac-HB (Heber Biotec, Cuba). Como unidad de aleatorización para la asignación al grupo de estudio o al grupo testigo se emplearon los 523 consultorios de los médicos de familia existentes en los municipios seleccionados. El estudio abarcó a toda persona de 20 a 64 años de edad de uno u otro sexo que residia en los municipios de Ranchuelo, Quemado, Santo Domingo, Encrucijada, Corralillo, Cifuentes y Camajuaní, en la provincia de Villa Clara, ubicada en la región central de Cuba, que aceptó participar voluntariamente en el ensayo. La vacunación se efectuó en los consultorios de los médicos de familia entre febrero y julio de 1998, con un intervalo de 6 semanas entre las dos dosis. El período de seguimiento fue de 12 meses. Se consideró positivo un caso si había recibido las dos dosis de la vacuna asignada y había enfermado de leptospirosis, con diagnóstico confirmado mediante métodos serológicos y microbiológicos, después de 21 días de aplicada la segunda dosis. Se calcularon la eficacia de la vacuna y el riesgo relativo (RR) de enfermar de leptospirosis después de la vacunación. Para el estudio de seguridad se escogió a dos personas al azar entre las personas vacunadas en cada uno de los consultorios que participaron en el estudio de eficacia. El seguimiento de las reacciones adversas locales y sistémicas lo realizaron los médicos de familia durante los siete días posteriores a la aplicación de cada dosis. El nivel de significación se fijó en 0,05 .

Resultados. En total se vacunó a 101832 personas, de las cuales 50354 (49,4\%) recibieron la vacuna contra la leptospirosis y 51478 (50,6\%) la vacuna de control. La eficacia de la vacuna vax-SPIRAL fue de 78,1\% (IC95\%: 59,2 a 88,3) y el RR de enfermar de leptospirosis de las personas vacunadas con respecto a las personas sin vacunar fue de 0,22 (IC95\%: 0,12 a

1 Instituto de Medicina Tropical Pedro Kourí, Ciudad de La Habana, Cuba. La correspondencia debe dirigirse a Raydel Martínez Sánchez, Instituto de Medicina Tropical Pedro Kourí, Autopista Novia del Mediodía km. 6.5, Apartado Postal 601, Maria- nao 13, Ciudad de La Habana. Cuba. Correo electrónico: raydel@ipk.sld.cu.

2 Instituto de Sueros y Vacunas Finlay, Ciudad de La Habana, Cuba.
3 Centro Provincial de Higiene y Epidemiología de Villa Clara, Santa Clara, Villa Clara, Cuba.

4 Dirección de Epidemiología, Ministerio de Salud Pública, Ciudad de La Habana, Cuba. 
0,41). El malestar general fue la reacción adversa sistémica más frecuente y el dolor espontáneo ligero en el sitio de la inyección el más frecuente de los efectos locales. En ambos casos estas reacciones fueron mayores en el grupo de estudio que en el grupo testigo ( $\mathrm{P}=0,003)$. No se produjeron reacciones adversas graves.

Conclusión. La vacuna cubana vax-SPIRAL se mostró segura y eficaz para el control de la leptospirosis, por lo que se recomienda su utilización para prevenir esta enfermedad en los grupos en riesgo de contraerla.

Leptospirosis, vacunas bacterianas, eficacia, Cuba.
La leptospirosis, una de las zoonosis de más amplia distribución geográfica en el mundo, se considera una enfermedad infecciosa emergente y constituye un problema de salud pública en muchos países en desarrollo por su alta letalidad (1-3).

En Cuba, esta enfermedad ha mostrado un comportamiento endémico y epidémico con características epidemiológicas cambiantes. Entre 1980 y 1990 aumentó ligeramente la incidencia a 6,0 × $10^{5}$ habitantes, entre $1991 \mathrm{y}$ 1995 se observó una etapa marcada por el brusco incremento del número de casos detectados $\left(25,6 \times 10^{5}\right.$ habitantes en 1994) y finalmente ocurrió una franca reducción de la incidencia entre 1996 y $2002\left(10,1\right.$ y $4,5 \times 10^{5}$ habitantes, respectivamente) $(4,5)$.

Los conocimientos acumulados acerca de la enfermedad y la forma de enfrentarla han servido de base a las autoridades cubanas del sector de la salud para elaborar el Programa Nacional de Prevención y Control de la Leptospirosis, puesto en vigor en 1981 (6). Según lo previsto en este Programa, en 1983 se comenzó a aplicar la vacuna antileptospirósica Vaccinum Leptospirosum (Instituto de Sueros y Vacunas, Stavropol, Rusia) a personas en mayor riesgo de contraer la infección en las áreas endémicas. Esta vacuna se aplicó por la vía subcutánea en dos dosis, la primera de 2,0 mL y la segunda de 2,5 $\mathrm{mL}$, con un intervalo de siete días.

En 1991 se dejó de importar esta vacuna y comenzaron los estudios para elaborar una vacuna cubana contra la leptospirosis con las cepas autóctonas de mayor circulación en el país. Estudios preclínicos han demostrado la potencia inmunógena y protectora de esta vacuna (7), mientras que según investigaciones realizadas en humanos mediante estudios controlados y con doble enmascaramiento, su administración no ha provocado reacciones adversas graves (8). Según un ensayo de fase II, la frecuencia de febrícula y malestar fue mayor en los que recibieron la vacuna cubana que en los que recibieron la vacuna de control Vaccinum Leptospirosum, pero la mayoría de los síntomas y signos desaparecieron en menos de 72 horas (9). La tasa de seroconversión fue de 29,0-34,2\% (10).

El presente estudio recoge los resultados del primer ensayo clínico para evaluar la eficacia (fase III) de la vacuna cubana contra la leptospirosis (vax-SPIRAL) aplicada en algunos municipios de la provincia de Villa Clara, Cuba, y aporta información adicional acerca de la seguridad de esta vacuna.

\section{MATERIALES Y MÉTODOS}

\section{Ensayo de eficacia}

Se realizó un ensayo de eficacia (fase III) controlado, aleatorizado (1:1) y con doble enmascaramiento de la vacuna cubana contra la leptospirosis vaxSPIRAL (Instituto Finlay, Cuba). Esta vacuna es una bacterina que contiene entre $50 \times 10^{6}$ y $80 \times 10^{6}$ células de Leptospira canicola, L. copenhageni y L. mozdok adsorbidas en $1 \mathrm{mg}$ de gel de hidróxido de aluminio en $0,5 \mathrm{~mL}$ de tampón fosfato salino con $0,05 \mathrm{mg}$ de tiomersal.

Como control se utilizó la vacuna recombinante contra la hepatitis B He-
berbiovac-HB (Heber Biotec, Cuba) que contiene $20 \mu \mathrm{g}$ de una proteína recombinante del antígeno de superficie del virus de la hepatitis $B$ adsorbidos en 0,25 mg de gel de hidróxido de aluminio en $0,5 \mathrm{~mL}$ de tampón fosfato salino con 0,025 mg de tiomersal.

La población escogida para el ensayo de eficacia de la vacuna se compuso de 131832 personas de 20 a 64 años de edad de uno $u$ otro sexo que residían en los municipios de Ranchuelo, Quemado, Santo Domingo, Encrucijada, Corralillo, Cifuentes y Camajuaní, en la provincia de Villa Clara, ubicada en la región central de Cuba, que aceptaron participar voluntariamente en el ensayo. El número de casos de infección por Leptospira en esos municipios era superior al del resto del país (de 41,8 a $112,5 \times 10^{5}$ habitantes) y presentaba una tendencia ascendente desde 1987, por lo que su población estaba expuesta a un mayor riesgo de contraer la enfermedad.

Se excluyó a las mujeres embarazadas y a aquellas personas que en el momento de la vacunación presentaban alguna enfermedad crónica descompensada o una infección aguda. También se excluyó del análisis a 11205 personas $(8,5 \%)$ que no cumplieron el esquema de vacunación previsto en el diseño de la investigación.

La vacunación se efectuó en los consultorios de los médicos de familia -que son las unidades básicas de organización de la asistencia médica en los municipios - con un intervalo de 6 semanas entre las dos dosis. El período de seguimiento fue de 12 meses, contados a partir del período de seroconversión de 21 días posteriores a la 
segunda dosis. La vacunación se realizó entre febrero de 1998 y julio de 1998 y el período de vigilancia se prolongó hasta agosto de 1999.

Como unidad de aleatorización se emplearon los 523 consultorios de los médicos de familia de los municipios seleccionados. Para garantizar el doble enmascaramiento se utilizaron códigos que identificaron los lotes de las dos vacunas empleadas en el estudio. El código fue secreto y se guardó en dos sobres que permanecieron sellados en la dirección del Instituto de Medicina Tropical Pedro Kourí - encargado del ensayo de fase III de la vacuna vaxSPIRAL - y en la del Instituto Finlay - productor de esa vacuna- hasta finalizar el período de vigilancia.

Una vez iniciado el estudio, en toda la provincia se puso en marcha un mecanismo de vigilancia epidemiológica activa y se notificaron todos los casos de leptospirosis, independientemente de los antecedentes de vacunación del paciente. A todos los casos que se presentaron en los consultorios del médico de familia, en las policlínicas o en los hospitales se les tomó una muestra de sangre para su estudio serológico y microbiológico.

Análisis estadístico. La eficacia de la vacuna se calculó mediante la fórmula descrita por Orenstein y colaboradores (11), basada en la comparación de las tasas de incidencia en personas vacunadas y sin vacunar, mediante la fórmula $E V=T N V-T V / T N V \times 100$, siendo $T N V$ la tasa de incidencia en los no vacunados y TV la tasa de incidencia en los vacunados.

Se empleó la prueba de ji al cuadrado para comparar las proporciones y se calculó el riesgo relativo (RR) de enfermar de leptospirosis como medida de asociación entre la vacunación y la aparición de la enfermedad (12). El nivel de significación se fijó en 0,05 y la potencia del ensayo en $80 \%$.

\section{Estudio de seguridad}

La muestra para el estudio de seguridad quedó conformada por dos personas escogidas al azar entre los vacunados de cada uno de los 523 consultorios de médicos de familia de los siete municipios escogidos. El tamaño de la muestra se determinó mediante el programa Epi Info versión 6 (13), de acuerdo con los resultados de estudios previos sobre la reactogenicidad de estas vacunas, según los cuales se podía esperar un $29 \%$ de reacciones adversas en el grupo de estudio $(8,9)$ y un $22 \%$ en el grupo testigo (14). El nivel de confianza establecido fue de $95 \%$ y el poder del estudio de $80 \%$.

La asignación de los consultorios al grupo de estudio (vacunados con la vacuna contra la leptospirosis vaxSPIRAL) o al grupo testigo (vacunados con la vacuna contra la hepatitis B Heberbiovac HB) se realizó de forma aleatoria. Los consultorios se estratificaron en urbanos y rurales, según su ubicación. Se generaron aleatoriamente dos grupos de consultorios de manera que fueran similares según su población y ubicación.

Cada médico de familia realizó el seguimiento de las dos personas vacunadas en su área de atención durante los 7 días posteriores a la aplicación de cada dosis y registró diariamente en los modelos de recogida de datos todas las reacciones adversas locales (eritema, induración, edema, dolor local, prurito, necrosis y absceso, entre otras) y sistémicas (malestar general, fiebre, febrícula, cefalea, lipotimia, náuseas, vómitos $\mathrm{y}$ exantema) detectadas mediante un interrogatorio y examen físico. La fiebre se definió como una temperatura corporal, constatada por aplicación de un termómetro en la axila, de $38{ }^{\circ} \mathrm{C}$ o más, y la febrícula como una temperatura corporal entre 37 y $38^{\circ} \mathrm{C}$, determinada por el mismo método. La induración y el eritema se midieron con reglas milimetradas.

Análisis estadístico. Se calculó el porcentaje de cada tipo de reacción observada después de la vacunación, tanto en el grupo de estudio como en el grupo testigo. Las diferencias entre proporciones se analizaron mediante la prueba de ji al cuadrado y la prueba exacta de Fischer, con un nivel de significación de 0,05.

\section{Criterios de diagnóstico}

Se consideraron positivos los casos si presentaban síntomas y signos compatibles con la leptospirosis, independientemente de los indicios epidemiológicos; si se aislaba el agente etiológico (Leptospira interrogans sensu lato); si en la segunda prueba serológica del paciente se observaba la seroconversión o un título de anticuerpos al menos cuatro veces mayor que en la prueba inicial; o si una muestra de suero en una dilución de 1:160 ó mayor reaccionaba positivamente a la prueba de hemaglutinación pasiva (6).

El diagnóstico serológico de los pacientes se realizó mediante microaglutinación con antígenos vivos de 13 serogrupos del complejo L. interrogans con una dilución inicial del suero de 1:10 (15). La hemaglutinación pasiva se realizó según el procedimiento descrito en el Programa Nacional de Prevención y Control de la Leptospirosis Humana (6).

Las muestras para el cultivo se tomaron antes de aplicar cualquier tratamiento al paciente. Para ello se inocularon dos tubos con medio de cultivo Ellinghausen modificado por Johnson y Harris (16), a los que se agregaron una y dos gotas de sangre, respectivamente. Estas muestras se trasladaron al Centro Provincial de Higiene y Epidemiología de Villa Clara, donde se incubaron hasta 40 días a $28-30{ }^{\circ} \mathrm{C}$ para investigar la presencia de Leptospira. Los aislamientos se enviaron al Laboratorio Nacional de Referencia de Leptospiras, en el Instituto de Medicina Tropical Pedro Kourí, para su caracterización serológica con antígenos vivos y sueros policlonales específicos para los 13 serogrupos patógenos de este microorganismo.

Se consideró que un caso era positivo si había recibido las dos dosis de la vacuna asignada y había enfermado de leptospirosis después de 21 días de aplicada la segunda dosis.

\section{Aspectos éticos del estudio}

Durante la investigación se cumplieron las pautas de buenas prácticas clí- 
nicas y se observaron los principios éticos establecidos para las investigaciones con seres humanos $(17,18)$. Se contó con el consentimiento informado de los participantes del estudio. Los voluntarios fueron informados por su médico de familia acerca de los objetivos de la investigación y de la posibilidad de ser asignados al grupo de estudio o al grupo testigo. Además, fueron informados del carácter investigativo del estudio, los beneficios esperados, los posibles riesgos, la duración de su participación en él, sus responsabilidades y los procedimientos de la investigación. El estudio fue aprobado por los comités de ética del Instituto de Medicina Tropical Pedro Kourí y del Instituto Finlay. La metodología de trabajo y el monitoreo de los datos del estudio de seguridad fueron supervisados por el Centro para el Control Estatal de la Calidad de los Medicamentos de Cuba (CECMED), que es la autoridad nacional reguladora designada por el Ministerio de Salud Pública de Cuba.

\section{RESULTADOS}

En el estudio participaron 101832 personas, o el equivalente de $77,2 \%$ de la población inicial posible (131 832). En total, 12524 personas $(9,5 \%)$ no se presentaron en el momento de la vacunación (se encontraban fuera de la localidad o tenían alguna enfermedad aguda) y $6271(4,8 \%)$ no aceptaron participar. Del análisis se excluyó a otras 11205 personas $(8,5 \%)$ que no cumplieron el esquema de vacunación previsto en el diseño de la investigación. No se presentaron reacciones adversas graves que obligaran a ningún paciente a abandonar el estudio.

De los participantes, $50354(49,4 \%)$ recibieron la vacuna contra la leptospirosis y $51478(50,6 \%)$ la vacuna de control.

\section{Cálculo de la eficacia}

Durante la vigilancia epidemiológica posterior a la vacunación se presentaron 68 casos confirmados de lep- tospirosis que cumplían con los criterios establecidos. De ellos, 12 casos pertenecían al grupo que recibió la vacuna contra la leptospirosis humana y 56 al grupo testigo. Las tasas de incidencia en cada grupo fueron de $2,38 \times$ $10^{4}$ y $10,89 \times 10^{4}$, respectivamente $(P<0,0001)$.

La eficacia específica de la vacuna en la población de los municipios seleccionados al año de su administración fue de 78,1\% (IC95\%: 59,2 a 88,3) y el RR de contraer leptospirosis en personas vacunadas con respecto a personas sin vacunar fue de 0,22 (IC95\%: 0,12 a 0,41).

\section{Reacciones adversas}

De los voluntarios vacunados que participaron en el estudio de seguridad, $950(90,8 \%)$ pudieron ser evaluados después de las dos dosis de la vacuna. Ninguno de los participantes manifestó efectos secundarios que justificaran hospitalizarlo o sacarlo del estudio.

La reacción sistémica encontrada con mayor frecuencia después de la primera dosis fue el malestar general (26,5\% en el grupo testigo frente a $34,2 \%$ en los vacunados con vax-SPIRAL; $P=0,01$ ) (figura 1). Aunque a raíz de la segunda dosis disminuyó en

FIGURA 1. Síntomas y signos generales manifestados durante los siete días posteriores a la vacunación con vax-Spiral. Primera dosis. Cuba, 1998

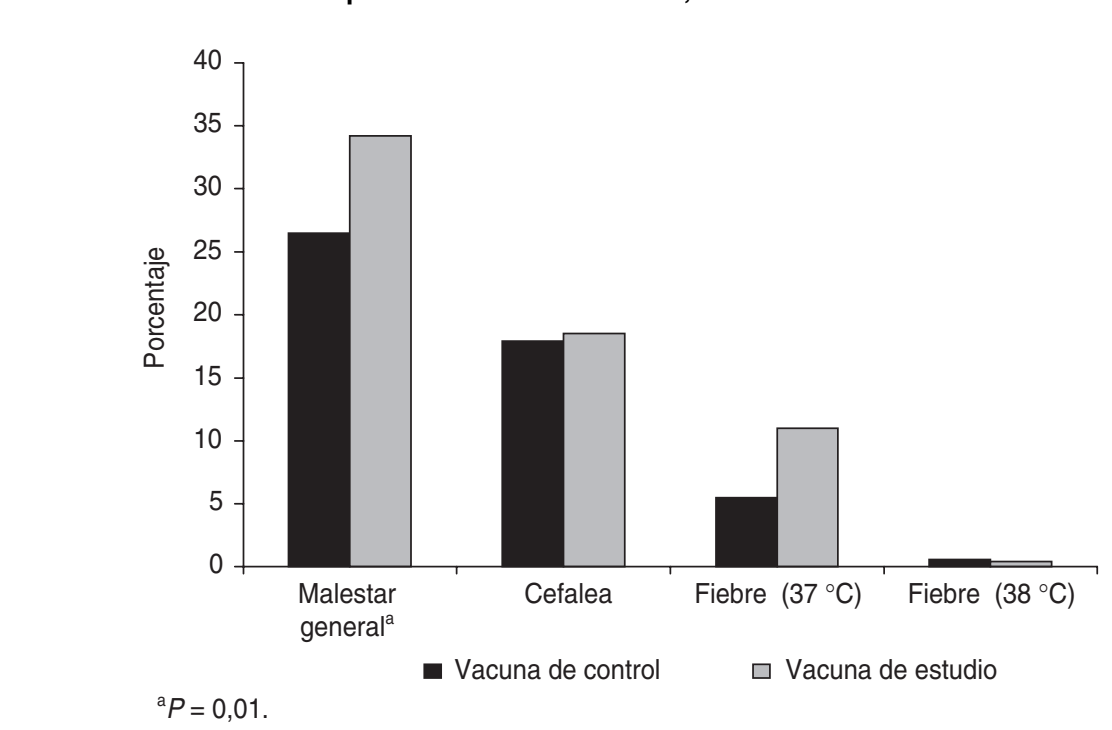

ambos grupos la frecuencia del malestar general, esta siguió siendo la reacción adversa más frecuente, con un número de casos significativamente mayor entre quienes recibieron la vacuna contra la leptospirosis que entre quienes recibieron la vacuna de control (20,3\% frente a $32,8 \%$, respectivamente; $P<0,0001$ ) (figura 2). Después de ambas dosis se produjo fiebre en un porcentaje muy pequeño de todos los vacunados, sin que se observara ninguna diferencia entre ambos grupos $(P=0,4)$.

De las reacciones adversas locales observadas después de la primera dosis, la más frecuente fue el dolor espontáneo ligero en el sitio de la inoculación, sin menoscabo funcional ni orgánico. Esta reacción se observó en ron la vacuna estudiada frente a $10,5 \%$ de los que recibieron la vacuna de control $(P=0,01)$ (figura 3). Después de la segunda dosis, estas cifras descendieron: se observó dolor local espontáneo en el sitio de la inyección en 12,5\% de los que recibieron la vacuna en estudio y en $8,3 \%$ del grupo testigo $(P=0,04)$ (figura 4). El resto de las manifestaciones locales se presentaron en el transcurso de las primeras 72 horas, con poca frecuencia y sin diferencias significativas entre los dos grupos. $16,1 \%$ de los participantes que recibie- 
FIGURA 2. Síntomas y signos generales manifestados durante los siete días posteriores a la vacunación con vax-Spiral. Segunda dosis. Cuba, 1998

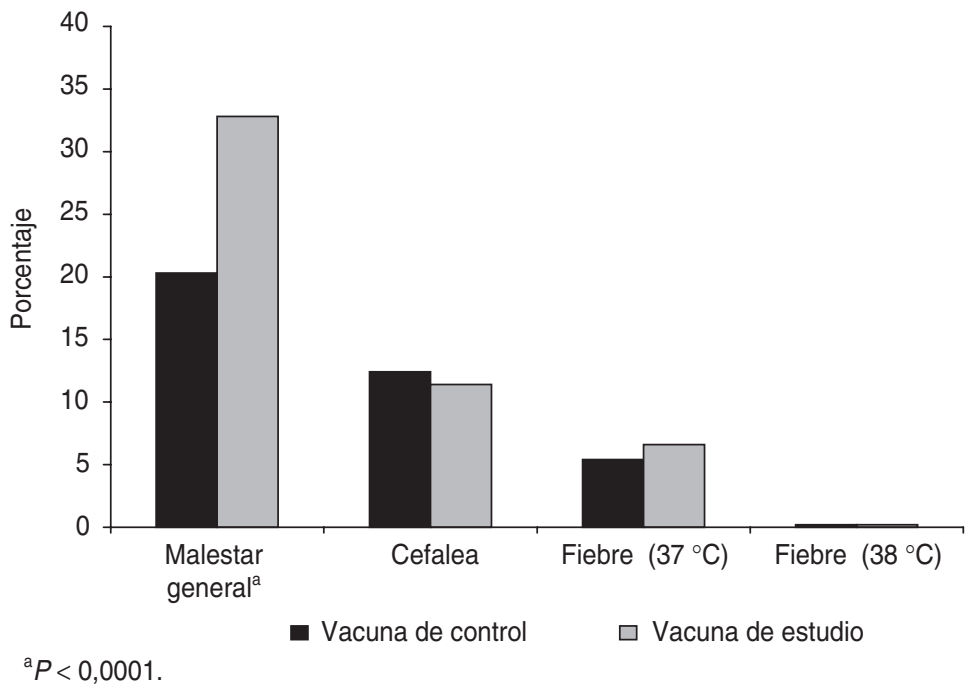

\section{DISCUSION}

Los resultados de este estudio permiten caracterizar la eficacia y seguridad de la vacuna cubana contra la leptospirosis humana en una población expuesta al riesgo de infección por residir en una zona con una alta incidencia de esta enfermedad. El diseño del estudio de eficacia (aleatorizado con doble enmascaramiento) evitó introducir sesgos en la selección de los voluntarios y contribuyó a garantizar la equivalencia de los dos grupos vacunados, lo que permitió llegar a resultados confiables.

Las primeras vacunas contra la leptospirosis, tanto para uso en humanos como en animales, consistían en preparaciones de células muertas por ac-

FIGURA 3. Síntomas y signos locales manifestados durante los siete días posteriores a la vacunación con vax-Spiral. Primera dosis. Cuba, 1998

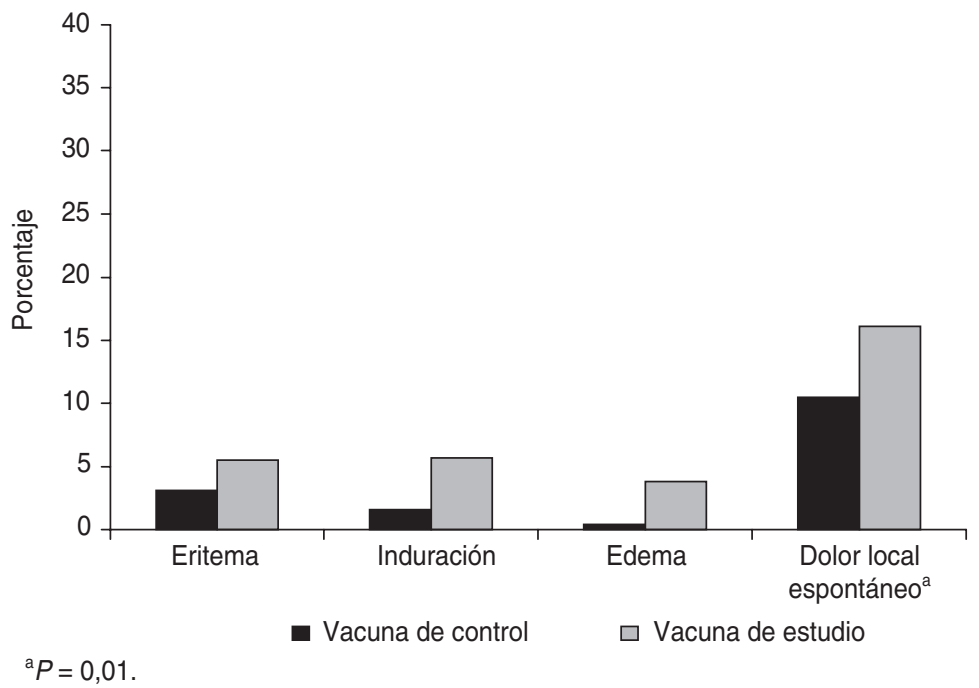

ción del calor o inactivadas con reactivos químicos, como la formalina. Estas células se inoculaban en medios de cultivo que contenían suero de origen animal, componente que solía causar reacciones adversas importantes (19, 20). La inmunización con vacunas polivalentes contra la leptospirosis se ha aplicado en países como China y Japón, donde la incidencia de esta enfermedad es alta entre las personas que se dedican al cultivo del arroz (21).

Se conocen pocos estudios de la eficacia de este tipo de vacuna y ninguno que se haya realizado a gran escala (22-24). En un estudio realizado en Israel en 1973 con una vacuna autóctona sin proteínas séricas aplicada a personas en riesgo se alcanzó un nivel de seroconversión de 57\% (22). Para que una vacuna sea eficaz no solo tiene que cumplir con los requisitos tecnológicos, sino que también se debe preparar a partir de cepas que respondan a los serotipos de mayor circulación en el área (25). La eficacia específica alcanzada por la vacuna aquí estudiada fue de $78,1 \%$, comparable con la notificada para vacunas de características similares en ensayos de eficacia realizados en China y Rusia $(21,23,26)$.

Los casos de leptospirosis detectados en los municipios donde se realizó el estudio afectaron fundamentalmente a la población que no había recibido la vacuna contra la leptospirosis (2,38 casos / 10000 habitantes en personas vacunadas frente a 10,89 casos/ 10000 habitantes en personas sin vacunar) $(P<0,0001)$. Este resultado es un indicador de la eficacia de la vacuna, ya que la disminución de la incidencia de una enfermedad después de la vacunación, cuando no se ha modificado el sistema de vigilancia, refleja la eficacia de la vacuna empleada (11). Durante todo el período de vigilancia del presente estudio no se realizaron otras intervenciones sanitarias que las establecidas en el Programa Nacional de Control de la Leptospirosis, las cuales ya se aplicaban de forma sistemática desde los primeros años de la década de 1980. La definición de un caso y los medios de confirmación del diagnóstico no sufrieron variaciones durante el período analizado. 
FIGURA 4. Síntomas y signos locales manifestados durante los siete días posteriores a la vacunación con vax-Spiral. Segunda dosis. Cuba, 1998

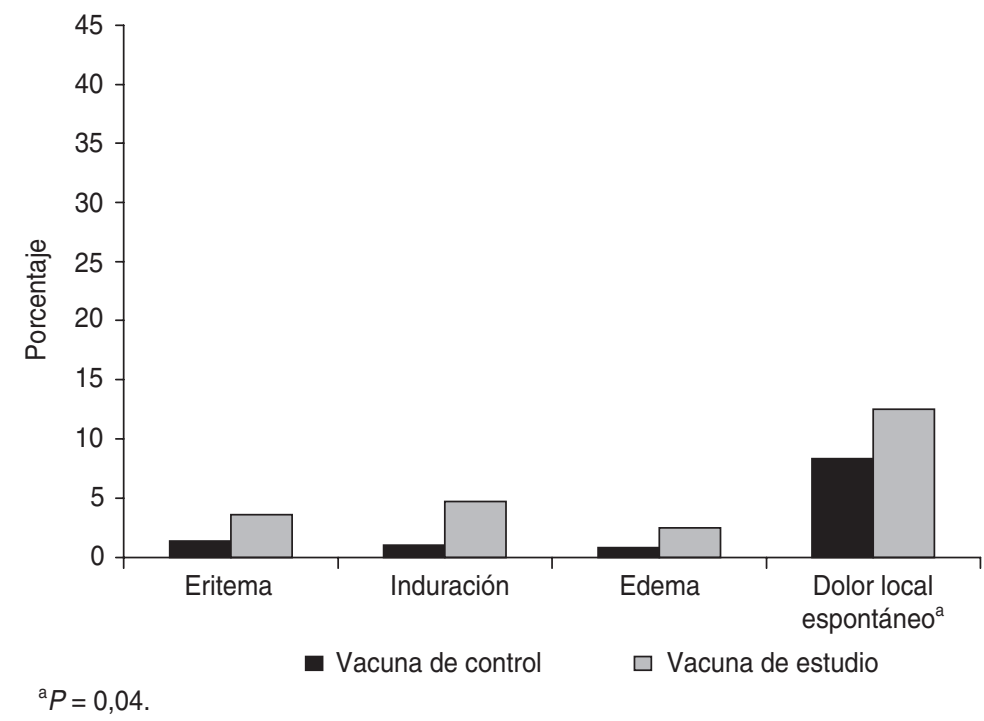
tan datos científicos que respaldan la utilidad de la vacuna vax-SPIRAL para la prevención de la leptospirosis en personas expuestas a un mayor riesgo de infección, por lo que se recomienda su utilización en los grupos en riesgo.

\section{Reacciones adversas} sas provocadas habitualmente por las vacunas son ligeras y de corta dura-
Los hallazgos de este estudio apor-

La mayoría de las reacciones adver-

ción. Estas pueden ser reacciones sistémicas, como fiebre, exantemas y linfadenopatías, o reacciones locales, como dolor, enrojecimiento y tumefacción en el sitio de la inyección. Tales reacciones generalmente no ocasionan daños permanentes (27).

El presente ensayo con doble enmascaramiento confirmó resultados anteriores en el sentido de que la vacuna antileptospirósica cubana vax-SPIRAL no produce reacciones sistémicas adversas graves y de que las reacciones locales son ligeras y de corta duración $(8,9)$. Tanto la vacuna antileptospiró-

\section{REFERENCIAS}

1. Bharti AR, Nally JE, Ricaldi JN, Matthias MA, Díaz MM, Lovett MA, et al. Leptospirosis: a zoonotic disease of global importance. Lancet Infect Dis. 2003;3(12):757-71.

2. Lomar AV, Diament D, Torres JR. Leptospirosis in Latin America: emerging and re-emerging diseases in Latin America. Infect Dis Clin. 2000;14(1):23-38.

3. Acha P, Szyfres SB. Zoonosis y enfermedades transmisibles comunes al hombre y los animales. 3. ${ }^{\mathrm{a}}$ ed. Washington, D.C.: Organización Panamericana de la Salud; 2001. (Publicación Científica y Técnica No. 580).

4. Cuba, Ministerio de Salud Pública. Cuadro epidemiológico nacional, 1987-2003. La Habana: Ministerio de Salud Pública; 2004.
5. Cruz de la Paz R. Leptospirosis. Rev Cubana Med Trop. 2002;54(1):6.

6. Cuba, Ministerio de Salud Pública. Programa pirosis Humana. La Habana: MINSAP; 1998.

7. González M, Naranjo M, Rodríguez Y, Bebelagua Y, Oliva R. Vacuna antileptospirósica trivalente adsorbida para uso humano. Primer ensayo evaluativo de reactogenicidad e inmunogenicidad en un grupo de voluntarios adultos. VacciMonitor. 1997;6(12):2-10.

8. Martínez R, Obregón AM, Pérez A, Baly A, Díaz M, Baró M, et al. Evaluación de la reactogenicidad e inmunogenicidad de la primera vacuna contra la leptospirosis humana. Rev Cubana Med Trop. 1998; 50(2):159-66. Nacional de Prevención y Control de la Leptos- sica rusa como la desarrollada en China - ambas sin adyuvante- ocasionan reacciones adversas moderadas, posiblemente debido a que contienen más componentes celulares que vaxSPIRAL $(21,28)$. En un estudio realizado en Israel con otra vacuna contra la leptospirosis — también de células enteras inactivadas y sin adyuvante- se observaron reacciones adversas leves, aunque hubo algunas reacciones alérgicas sistémicas y cutáneas atribuidas a los anticuerpos circulantes o al efecto irritativo de la propia vacuna o de la formalina utilizada para la inactivación celular (24).

La menor reactogenicidad de la vacuna antileptospirósica vax-SPIRAL podría deberse a las particularidades del proceso de inactivación celular y del tratamiento posterior aplicado durante su elaboración, que conservan sin grandes daños los principales antígenos celulares, pudiéndose así reducir la cantidad de antígeno celular por dosis (7).

En cuanto al adyuvante, aunque este puede empeorar los síntomas locales, las reacciones adversas que se presentan son similares a las observadas con vacunas sin adyuvante, tanto por su frecuencia como por su intensidad, y fueron bien toleradas por los voluntarios.

En resumen, la vacuna cubana vaxSPIRAL se mostró segura y eficaz para el control de la leptospirosis, por lo que se recomienda su utilización para prevenir esta enfermedad en los grupos en riesgo.
9. Martínez R, Pérez A, Baly A, Baró M, Menén$\operatorname{dez} R$, Ruiz A, et al. Reactogenicidad de la vacuna cubana trivalente contra la leptospirosis humana en un ensayo clínico de fase II. VacciMonitor. 2001;10(1):1-5.

10. Obregón AM. Estudio serológico de las fases I y II de la primera vacuna cubana contra la leptospirosis humana. [tesis de maestría en Bacteriología y Micología]. Ciudad de La Habana: Instituto de Medicina Tropical Pedro Kourí; 1999.

11. Orenstein WA, Roger HB, Alan BH. Assessing vaccine efficacy in the field: further observations. Epidemiol Rev. 1988;10:212-40.

12. Collimon M. Fundamentos de Epidemiología Medellín, Colombia: Díaz de Santos; 1993. 
13. Centers for Disease Control and Prevention. Epi Info versión 6.04 [programa de computación]. Atlanta: CDC; 1996.

14. Pedroso P, Díaz M. Comportamiento reactogénico de la vacuna cubana contra la hepatitis $\mathrm{B}$ en niños física y mentalmente discapacitados. Rev Cubana Med Gen Integr. 2000;16(5): 423-9.

15. World Health Organization. Human leptospirosis: guidance for diagnosis, surveillance and control. Geneva: WHO; 2003.

16. Ellinghausen HC, Mc Cullough WG. Nutrition of Leptospira pomona and growth of 13 other serotypes: fractionation of oleic albumin complex and a medium of bovine albumin and polysorbate 80. Am J Vet Res. 1965;26: 45-51.

17. Consejo de Organizaciones Internacionales de Ciencias Médicas. Normas éticas internacionales para las investigaciones biomédicas en sujetos humanos. Cuad Prog Reg Bioética. 1996;(3):121-31.

18. Stark NJ. World Medical Association Declaration of Helsinki. Ethical principles for medical research involving human subjects [sitio en Internet]. Edimburgo, Escocia 2000. Hallado en: http://www.wma.net/. Acceso el 24 de febrero 2004.
19. Christopher WL, Adler B, Faine S. Immunogenicity of leptospiral vaccines grown in protein-free medium. J Med Microbiol. 1982; 15:493-501.

20. Branger $\mathrm{C}$, Sonrier $\mathrm{C}$, Chatrenet $\mathrm{B}$, Klonjkowski B, Ruvoen-Clouet N, Aubert A, et al Identification of the hemolysis-associated protein 1 as a cross-protective immunogen of Leptospira interrogans by Adenovirusmediated vaccination. Infect Immunol. 2001; 69(11):6831-38.

21. Chen TZ. Development and present status of leptospiral vaccine and technology of production of the vaccine in China. Ann Immunol Hung. 1986;26:125-51.

22. Shenberg E, Torten $M$. A new leptospiral vaccine. I. Development of a vaccine from leptospira grown in a chemically defined medium. J Infect Dis. 1973;128:642-6.

23. Iagovkin EA, Kostina NI, Vachaev BF, Romantsova T, Kondratenko VF, Bunin IE, et al. The improvement of immunobiological preparations against leptospirosis. An experimental study of a new concentrated purified vaccine against icterohemorrhagic leptospirosis for human immunization. Zh Mikrobiol Epidemiol Immunobiol. 1990;2:47-51.
24. Torten M, Shenberg E. A new leptospiral vaccine for use in man. II. Clinical and serologic evaluation of a field trial with volunteers. J Infect Dis. 1973;128:647-51.

25. Sonrier C, Branger C, Michel V, RuvoenClouet N, Ganiere JP, André-Fontaine G. Evidence of cross-protection within Leptospira interrogans in an experimental model. Vaccine. 2000;19(1):86-94.

26. Yan Y, Chen Y, Liou W, Ding J, Chen J, Zhang $\mathrm{J}$, et al. An evaluation of the serological and epidemiological effects of the outer envelope vaccine to leptospira. J Chin Med Assoc. 2003; 66(4):224-30.

27. Ehreth J. The global value of vaccination. Vaccine. 2003;21:596-600.

28. Cruz de la Paz R, Rodríguez P, López C, Atienzar E, Abreus J, Aldama F. Reactogenicidad de la vacuna antileptospirósica en Cuba. Rev Cub Hig Epidemiol. 1986;24(4):407-12.

Manuscrito recibido el 23 de julio de 2003. Aceptado para publicación, tras revisión, el 11 de marzo de 2004

ABSTRACT Objectives. To evaluate the efficacy of vax-SPIRAL, a Cuban vaccine against leptospirosis, and to provide additional information concerning the safety of this vaccine (which was developed by Cuba's Finlay Institute).

Efficacy and safety

Methods. This phase III efficacy trial of vax-SPIRAL was controlled, randomized, and double blind. The control vaccine used for the trial was Heberbiovac-HB (Heber Biotec, Cuba), a recombinant hepatitis B vaccine. The randomization unit for allocating persons to the study group or the control group were 523 family physician offices in the selected municipalities. The study covered the entire population of males and females from 20 to 64 years old who voluntarily agreed to participate, from the municipalities of Ranchuelo, Quemado, Santo Domingo, Encrucijada, Corralillo, Cifuentes, and Camajuaní, which are in the province of Villa Clara, in the central region of Cuba. The vaccinations were given in the physicians' offices between February and July 1998, with an interval of 6 weeks between the two doses. The follow-up period was 12 months. A case was considered positive if a person who had received the two doses of the vaccine became ill with leptospirosis more than 21 days after receiving the second dose, with the diagnosis confirmed through serological and microbiological methods. We calculated the efficacy of the vaccine and the relative risk of becoming ill with leptospirosis after the vaccination. For the safety study, two persons were chosen at random from among the individuals vaccinated at the office of each physician participating in the study. Follow-up of local and systemic adverse reactions was carried out by the family physicians during the seven days after the application of each dose. The level of statistical significance was set at 0.05 .

Results. A total of 101832 persons were vaccinated, with 50354 of them (49.4\%) receiving the leptospirosis vaccine and 51478 of them (50.6\%) receiving the control vaccine. The efficacy of the vax-SPIRAL vaccine was $78.1 \%$ (95\% confidence interval (CI): $59.2 \%$ to $88.3 \%$ ), and the relative risk of becoming ill with leptospirosis after receiving the leptospirosis vaccine was 0.22 (95\% CI: 0.12 to 0.41 ). General discomfort was the most frequent systemic adverse reaction, and mild spontaneous pain at the injection site was the most frequent local effect. The local and systemic adverse reactions were both more frequent in the study group than in the control group $(P=0.003)$. There were no serious adverse events.

Conclusion. The vax-SPIRAL vaccine proved to be safe and efficacious for leptospirosis control. The vaccine is recommended for use in preventing this disease among groups at risk of contracting it. 